\title{
A Survey and Analysis on Scheduling Algorithms in IEEE 802.16e (WiMAX) Standard
}

\author{
Swaroop R.Puranik \\ Department of Computer Science \\ and Engineering, BVBCET \\ Hubli, India
}

\author{
Vijayalakshmi M \\ Linganagouda Kulkarni, Ph.D \\ Department of Computer Science Department of Computer Science and \\ and Engineering, BVBCET \\ Hubli, India
}

\begin{abstract}
Wireless Man or WiMAX, where WiMAX stands on Worldwide Interoperability for Microwave Access, is one of the latest technologies in the Wire-Less World. The main goal of WiMAX is to deliver wireless communications with quality of service in a secured environment. Unlike wireless LANs, WiMAX networks incorporate several qualities of service (QoS) mechanisms at the Media Access Control (MAC) level for guaranteed services for data, voice and video. Its Mobility feature makes it different from the other IEEE 802.16 protocols which was based on Static WiMAX and provided the Wireless communication at fixed locations. This paper deals with the key features of the IEEE 802.16e scheduling algorithms at MAC layer, their classification and their survey in which several authors have already done the research in this field. In addition to ensuring feasible algorithm complexity and system scalability, the purpose of scheduling algorithms also includes optimal usage of resources, to assure what the QoS guarantees, to maximize good throughput and to minimize power consumption.
\end{abstract}

\section{General Terms}

Cross layer scheduling, WRR, WFQ.

\section{Keywords}

IEEE 802.16e, WiMAX, QoS, MAC.

\section{INTRODUCTION}

IEEE 802.16 a set of telecommunications technology standards aims at providing wireless access over long distances in a variety of ways - from point-to-point links to full mobile cellular type access . IEEE 802.16e also called WirelessMAN, covers a metropolitan area of several kilometers. WiMAX networks, a connection-oriented technology does not include multimedia data, because IEEE 802.16e is not present in current profiles of the WiMAX forum [1].It is estimated that a WiMAX base station can provide broadband wireless access in range up to 30 miles $(50 \mathrm{~km})$ for fixed stations and 3 to 10 miles $(5$ to $15 \mathrm{~km})$ for mobile stations with a maximum data rate of up to $70 \mathrm{Mbps}$ [1] \& [2] compared to 802.11a with 54 Mbps up to several hundred meters, EDGE (Enhanced Data Rates for Global Evolution) with $384 \mathrm{kbps}$ to a few km, or CDMA2000 (CodeDivision Multiple Access 2000) with 2 Mbps for a few km.

Since 2001, a number of variants of these standards have been issued and are still being developed. Like other standards, the specifications also constitute several competing proposals and contain many other features and mechanisms. The Worldwide Interoperability for Microwave Access Forum or WiMAX Forum [2] is a group of 400+ networking equipment vendors, service providers, component manufacturers and users who will decide which of the numerous options that are allowed in the IEEE 802.16 e standards should be implemented so that equipment from various vendors will inter-operate the fundamental requirements of next generation OFDMA based wireless mobile communication systems which consist on the cross-layer scheduling and resource allocation mechanism. There are several new issues raised due to the interference between the available accesses as the growth of network access technologies increase.

\subsection{Architecture of IEEE 802.16e}

The Architecture of IEEE 802.16e mainly contains two layers MAC and PHY as shown in Fig 1. MAC layer is further divided in to three layers CSL (Convergence Sub layer), MAC Common Part Sub layer and Security Sub layer or SSL.

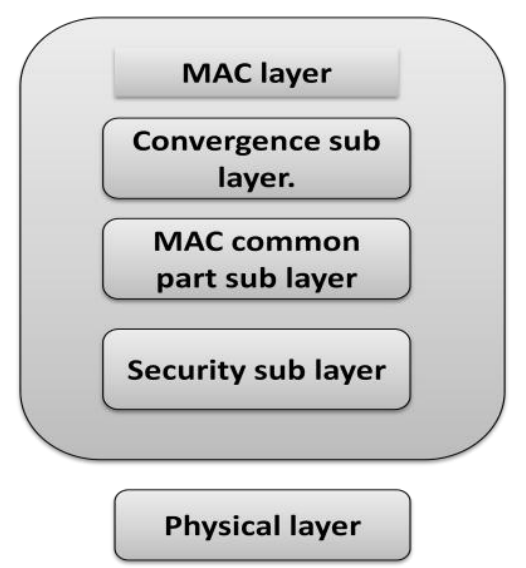

Fig 1: Architecture of IEEE 802.16e

Convergence Sub layer [2] receives the data from higher layer and forwards to CPS (Common Part Sublayer). The Convergence layer also sorts the incoming MACSDUs (Service Data Unit) by the connections to which they belong. Next Sub layer of MAC layer is CPS layer. In this layer, MAC protocol data units (PDUs) are constructed, connections are established and bandwidth is managed i.e. here Bandwidth and Connection Management are defined. CSL is tightly integrated with the Security sub layer. Last Layer of MAC Layer is SSL (Security Sub Layer).The Security sub layer addresses the authentication, establishment of keys and encryption. It exchanges MAC PDUs with the Physical layers. SSL defines two protocols Encapsulation and PKM Protocol whereas physical layer is responsible for receiving and transmitting MAC frames.

\subsection{QoS Services Classes}

In terms of guaranteed services, WiMAX includes several Quality of Service (QoS) mechanisms at the MAC (Media Access Control) layer. Typically, the QoS support in wireless networks is much more challenging than that in wired networks because the characteristics of the wireless link are highly variable and unpredictable both on a time-dependent basis and a location dependent basis. With a longer distance, multipath and fading effects are also put into consideration. To meet QoS requirements especially for voice and video transmission, with the delay and jitter constraints the key issue is how to allocate resources among the users and not only to achieve those 
constraints but also to maximize throughput, to minimize power consumption while keeping feasible algorithmic complexity and ensuring system scalability. [2] IEEE 802.16 standard does not specify any resource allocation mechanisms or admission control mechanisms.

Table 1: QoS service class in IEEE 802.16

\begin{tabular}{|l|l|}
\hline Qos Service Class & \multicolumn{1}{|c|}{ Description } \\
\hline $\begin{array}{l}\text { Unsolicited Grant } \\
\text { Service (UGS) }\end{array}$ & $\begin{array}{l}\text { Supports CBR services such as T1/E1 } \\
\text { emulation and VoIP without silence } \\
\text { suppression }\end{array}$ \\
\hline $\begin{array}{l}\text { Real-Time Polling } \\
\text { Service (rtPS) }\end{array}$ & $\begin{array}{l}\text { Supports real time services with variable } \\
\text { size data on a periodic basis, such as } \\
\text { MPEG and VoIP with silence suppression }\end{array}$ \\
\hline $\begin{array}{l}\text { Non-Real-Time } \\
\text { Polling Service } \\
\text { (nrtPS) }\end{array}$ & $\begin{array}{l}\text { Supports non real time services that } \\
\text { require variable size data grant bursts on a } \\
\text { regular basis, such as FTP }\end{array}$ \\
\hline $\begin{array}{l}\text { Best Effort } \\
\text { (BE) }\end{array}$ & $\begin{array}{l}\text { For application that do not require QoS, } \\
\text { such as web-surfing }\end{array}$ \\
\hline
\end{tabular}

The QoS traffic classes are as follows:

- Unsolicited grant service (UGS) supports constant bit rate $(\mathrm{CBR})$ or fixed throughput connections.

- Real-time polling service (rtPS) provides guarantees on throughput and latency, but with greater tolerance on latency relative to UGS.

- $\quad$ Nonreal-time polling service (nrtPS) provides guarantees in terms of throughput.

- Best effort (BE) service provides no guarantees on delay or throughput.

The purpose of this paper is to both provide a survey of recently proposed scheduling algorithms and give detailed information about WiMAX characteristics that need to be considered in developing a scheduler. In the second section we discuss about taxonomy of the scheduling algorithms and we will describe implemented scheduling algorithms and its obtained results based on its taxonomy. In the third section we will compare and analyze the results obtained from various papers based on QoS parameter like throughput, delay, jitter and fairness. Also in this section, we analyze which schedulers are best suited for various classes on different situation. In the fifth and final section we conclude about the analyzed results.

\section{TAXONOMY OF SCHEDULING ALGORITHM}

Scheduling algorithms are implemented at both the BS and SSs. In this paper the scheduling algorithms are defined in intra class schedulers and a scheduler at the SS is required to distribute the bandwidth allocation from the BS among its connections. A scheduling algorithm for the uplink traffic is faces challenges that are not faced by an algorithm for the downlink traffic. An uplink scheduling algorithm does not have all the information about the SSs such as the queue size. An uplink algorithm at the BS has to coordinate its decision with all the SSs where as a downlink algorithm is only concerned in communicating the decision locally to the BS. Figure 2 shows the taxonomy of the scheduling algorithms. Based on the comprehensive survey [8], the scheduling algorithms are classified into 3 categories:

- Homogenous scheduling algorithm

- Hybrid scheduling algorithm
- Opportunistic scheduling algorithm

In Homogenous scheduling algorithms individual algorithms are designed and implemented. Algorithms in this category do not address the issue of link channel quality.

In Hybrid scheduling algorithms they are designed with two or more homogenous schedulers to form a hybrid scheduler and these legacy scheduling algorithms in an attempt to satisfy QoS requirements of the four scheduling services. An important aspect of algorithms in this category is the overall allocation of bandwidth among the scheduling services.

In Opportunistic scheduling algorithms primary focus is on exploiting the variability in channel conditions in WiMAX.

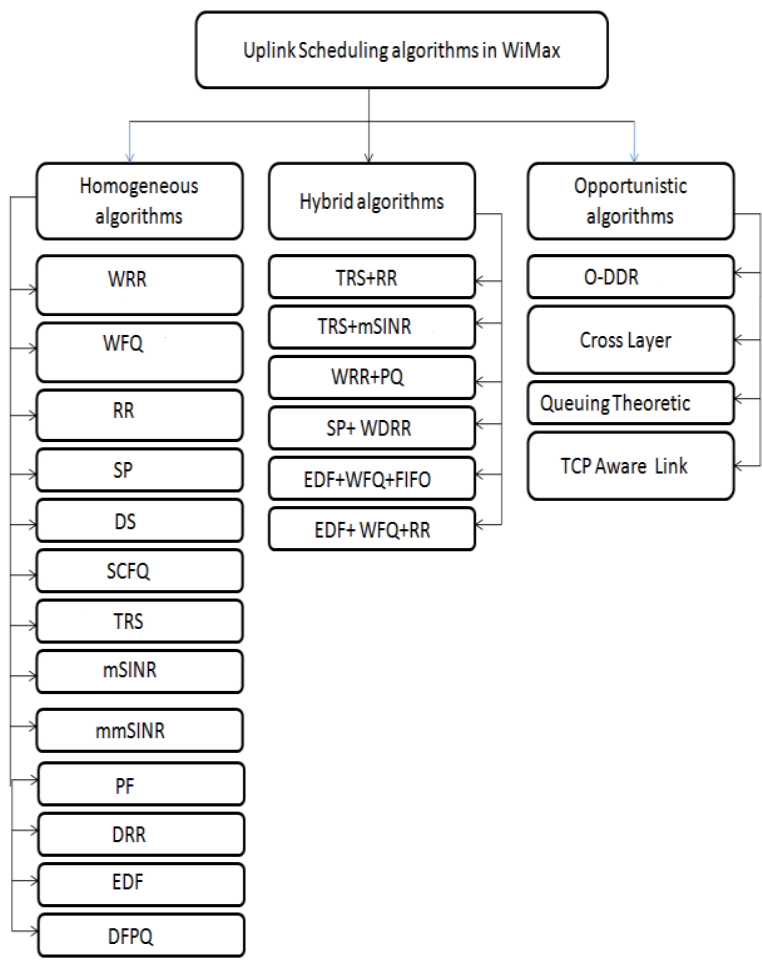

Fig 2: Taxonomy of Scheduling Algorithms

A simplified diagram of the scheduler in the standard IEEE 802.16e is illustrated in the following Figure 3:

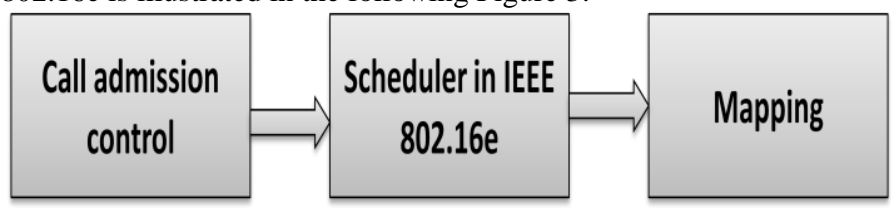

Fig 3: A simplified diagram of the scheduler

In CAC block any one of the bandwidth distributing mechanisms can be adopted between service classes and we assume that all connections accepted in the system are the result of applying this CAC strategy. In Scheduler block we choose the appropriate scheduler to schedule the packets for up-link/downlink. In mapping block the packets are directed towards the physical layer.

\subsection{Homogenous schedulers}

\subsubsection{Weighted Round Robin (WRR)}

It is a homogenous scheduling algorithm and its complexity is $\mathrm{O}$ (1). In [13],[14],[20] \&[21] WRR procedure, packets are categorized into different service classes and then assigned to a queue that can be assigned different percentage of bandwidth 
and served based on Round Robin order as shown in Figure 4. This algorithm address the problem of starvation by guarantees that all service classes have the ability to access at least some configured amount of network bandwidth. [25] Figure 4 demonstrates the WRR:

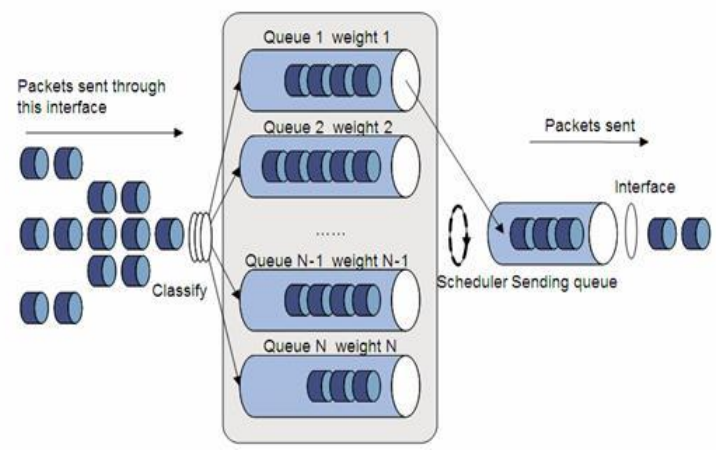

Fig 4: [25] WRR Scheduling Algorithm

[8] WRR algorithm indicates low average throughput of ertPS SSs, since the difference between MRTR and MSTR is also large, very high average delay for the ertPS class except when the concentration of ertPS SSs is the highest and poor performance when the packet size of the traffic is large. This behavior is indicated by the low average throughput of rtPS and nrtPS SSs, even under high concentration of rtPS and nrtPS SSs. [13]For rtPS QoS class the WRR always maintains almost high fairness, this is due to some real time packets rtVR connections are dropped under high burstiness and thus the throughput of rtVR decreases. [14] WRR does perform well compared to WFQ in queue management and resource utilization since it always maintains almost high fairness. [16] The delay for the two real-time classes (UGS \& rtPS) increases with time until exceeding the maximum delay limitation as required by their QoS latency parameters. [20] WRR outperformed the rest scheduling algorithms by producing the highest rate of throughput of data packet in the network. It is noted that WRR technique shows the most favorable results as the average jitter has low reading $(0.136 \mathrm{~s})$. [21] Says WRR is very useful algorithm for which the scheduler uses it only for one time. But when the case of hierarchy of WRR then it is challenging task to use because the peer-connection QoS requirements must be translated into scheduler at each level and when the network is dynamic, buffer length \& trade-off between the throughput and queue delay is difficult to control. [24] WRR schedulers provide a better mean sojourn time while delivering fewer data packets than the mSIR and TRS+mSIR schedulers. But TRS+RR scheduler performed better than WRR.

\subsubsection{Weighted Fair Queuing (WFQ)}

It is a homogenous scheduling algorithm and its complexity is $\mathrm{O}$ (N). In [14] Weighted Fair Queuing each flow are assigned different weight to has different bandwidth percentage in a way ensures preventing monopolization of the bandwidth by some flows providing a fair scheduling for different flows supporting variable-length packets by approximating the theoretical approach of the generalized processor sharing (GPS) system that calculates and assigns a finish time to each packet. [8] WFQ algorithm indicating low average throughput of ertPS SSs, since the difference between MRTR and MSTR is also large. When the concentration of SSs of the nrtPS class is high, the fairness among SSs of the ertPS class under the WFQ algorithm is the lowest. WFQ allocates bandwidth to the SSs based on their MRTR. This algorithm indicates a high average delay for the ertPS SSs when their concentration is low. The increase in average delay of SSs results in an increase in packet loss, although the relationship between average delay and packet loss is not as explicit it may result in a decrease in the average delay. [25]Figure 5 demonstrates the WFQ algorithm:

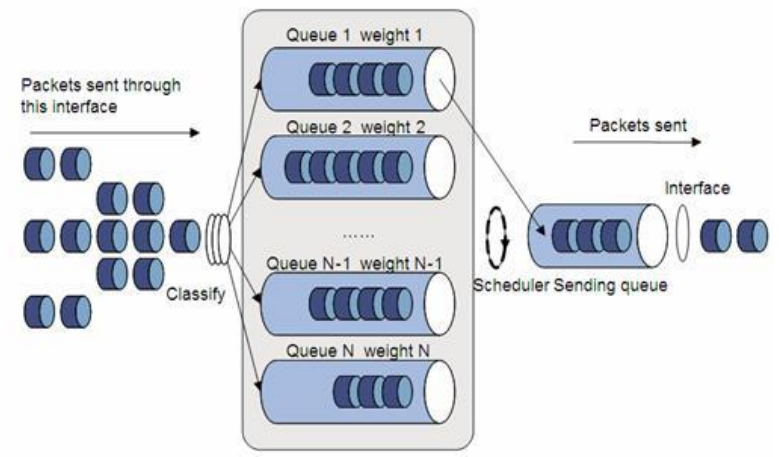

Fig 5: [25] WFQ Scheduling Algorithm

This is because the average delay does not include the delay of dropped packets. [10] Proposes a scheduler uses WFQ as the downlink as well as the uplink scheduling algorithm. [14] WFQ does not perform well compared to WRR and RR in queue management and resource utilization. [20] Showed the best performance as the average end-to-end time delay had the lowest reading, but it failed to perform when the number of MS became more than 40. It is noted that WFQ technique shows the most favorable results as the average jitter has low reading $(0.136 \mathrm{~s})$. [20], [24] it achieves the same amount of end-to-end delay time for the class BE and nrtPS. It is also noted that BE achieves the shortest amount of end-to end delay time for the Diff-Serve, WRR, SP. The WFQ algorithm results in superior performance compared to the WRR algorithm in the presence of variable size packets.

\subsubsection{Round Robin (RR)}

Round Robin as a scheduling algorithm is the most basic and least complex scheduling algorithm. It has a complexity value of $\mathrm{O}$ (1) [14]. RR is the best scheduling algorithms with queue management and resource utilization than any other scheduling algorithms like WFQ, WRR, DS etc. [20] It dominated other algorithms when the number of MSs became more than 50and the most efficient in terms of overall throughput $125 \mathrm{Kbps}$. RR algorithm was the best in terms of packet latency (Jitter). It is noted that RR technique shows the most favorable results as the average jitter has low reading $(0.124 \mathrm{~s})$. [24] The RR scheduler provide a better mean sojourn time while delivering fewer data packets but it is not performed in case of the real time streaming video.

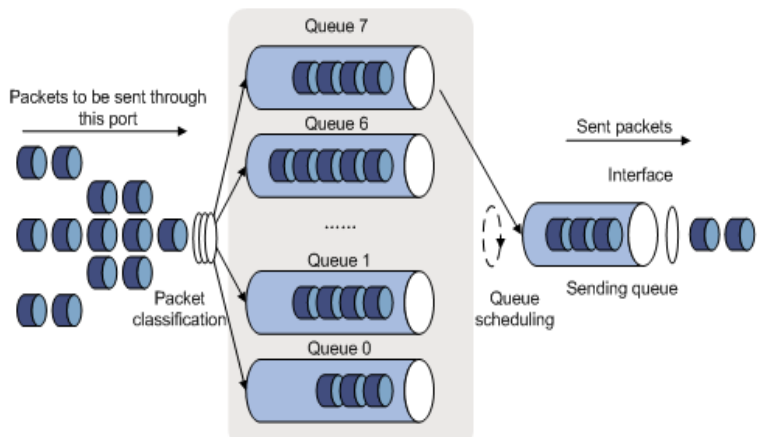

Fig 6: [25] RR Scheduling Algorithm 


\subsubsection{Earliest deadline first (EDF)}

It is a work conserving algorithm originally proposed for realtime applications in wide area networks and its complexity is $\mathrm{O}$ (N). The algorithm assigns deadline to each packet and allocates bandwidth to the SS that has the packet with the earliest deadline. Deadlines can be assigned to packets of a SS based on the SS's maximum delay requirement. [14]The EDF algorithm is suitable for SSs belonging to the UGS and rtVR scheduling services, since SSs in this class have stringent delay requirements. Since SSs belonging to the nrtVR service do not have a delay requirement, the EDF algorithm will schedule packets from these SSs only if there are no packets from SSs of UGS or rtVR class. From [8] the EDF algorithm schedules SSs based on their delay requirements only, the average throughput will be low. This will also reflect a low average throughput of rtPS SSs. The EDF algorithm indicates a lower average throughput for the nrtPS class when the concentration of ertPS or rtPS SSs is the highest since the algorithm provides strict priority to SSs with delay requirements (ertPS and rtPS SSs). A high average delay for the ertPS SSs when their concentration is low. This behavior is due to a low threshold assigned for the ertPS class. While the fairness of EDF [13] algorithm is the worst among the (WFQ, WRR, RR, TRS) algorithms. This is due to the fact that some real time packets rtVR connections are dropped under high burstiness, and thus the throughput of rtVR decreases.

\subsubsection{Strict-Priority $(S P)$}

In Strict-Priority algorithm [20], the selection order is based on the priority of weight order. The packets are first categorized by the scheduler depending on the quality of service (QoS) classes and then allocated into different priority queues. The algorithm services the highest priority queue until it is empty, after which, it moves to the next highest priority queue. Thus, strict-priority algorithm may not be suitable in WiMAX network. This is because there is no compensation for inadequate bandwidth. Also this technique is only appropriate for low-bandwidth serial lines that currently uses static configuration which does not automatically adapt to changing network requirements. Finally, this process may result in bandwidth starvation for the low priority QoS classes whereby the packets may not even get forwarded and no guarantee is offered to one flow. [14] shows that for BE and nrtPS traffic class almost had no traffic because the Strict-Priority scheduler caused bandwidth to be starved for low priority traffic types, the higher priority traffic had a higher throughput and the lowest priority traffic had low throughput. [20] SP produce almost the same amount of overall average for the throughput $110 \mathrm{Kbps}$.

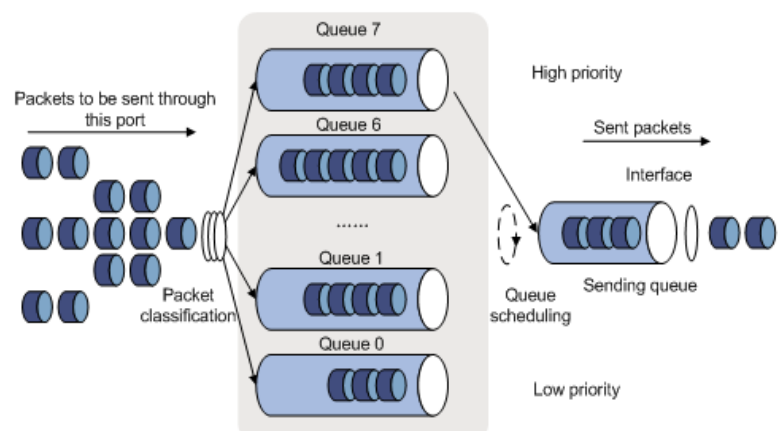

Fig 7: [25] SP Scheduling Algorithm

\subsubsection{Temporary Removal Scheduler}

The Temporary Removal Scheduler (TRS) scheduler [6] involves identifying the packet call power, depending on radio conditions, and then temporarily removing them from a scheduling list for a certain adjustable time period TR. In poor radio conditions, the whole process could be repeated up to $\mathrm{L}$ times at the end of which, the removed packed is added to the scheduling list, independently of the current radio channel condition. Results show that [13] if we consider latency as a function of rtVR+nrtVR traffic load, the TRS scheduler provides a decrease in the latency.

\subsubsection{Maximum Signal to Interference Ratio}

The scheduler mSIR (Maximum Signal to Interference Ration) is based on the allocation of radio resources to subscriber stations which have the highest SIR. [13] This scheduler allows a highly efficient utilization of radio resources. However, with the mSIR scheduler, the users with a SIR (Signal to Interference Ratio) that is always small may never be served. . If we consider [13] the latency as a function of rtVR traffic load it is verified that the mSIR scheduler provides a decrease in the latency. [14] TRS can be combined with the mSIR scheduler. From [24], the mSIR scheduler provides high throughput with a good SIR. From [18] the author proposed an algorithm and compared with mSIR and $\mathrm{PF}$. The delay stays low for proposed algorithm regarding the other algorithms even when the number of users increase, since in proposed cross-layer algorithm QoS of each SF is considered more than others and as ertPS service classes are more sensitive to delay constraints, takes more transmission opportunities than other types of SFs. MAX-SNR doesn't take into account the type of service flows and schedules the connections which have the best channel first. MAX-SNR with the most through-put value, it schedules connections without considering the quality of ser-vices and selects the connection with the best SNR for transmission. The spectral efficiency of the MAX-SNR scheme increases with respect to the number of users. But in proposed algorithm both the SNR and the QoS constraints are taken into account to guarantee the required QoS performance.

\subsubsection{Self-Clocked Fair (SCF) Queuing}

It is an efficient queuing scheme which satisfies the quality of services (QoS) in broadband implementation. It adopts the concept of an internally generated virtual time as the index of work in progress. The SCFQ algorithm can accomplish easier implementation and it can maintain the fairness attribute in virtual time function. [20] There is much difference in terms of the average end-to-end delay time among RR, SCF and WRR. But SCF produced almost the same amount of overall average for the throughput $110 \mathrm{Kbps}$ which is better than WRR. Figure 8 from [25] illustrates the work progress of SCF scheduler.

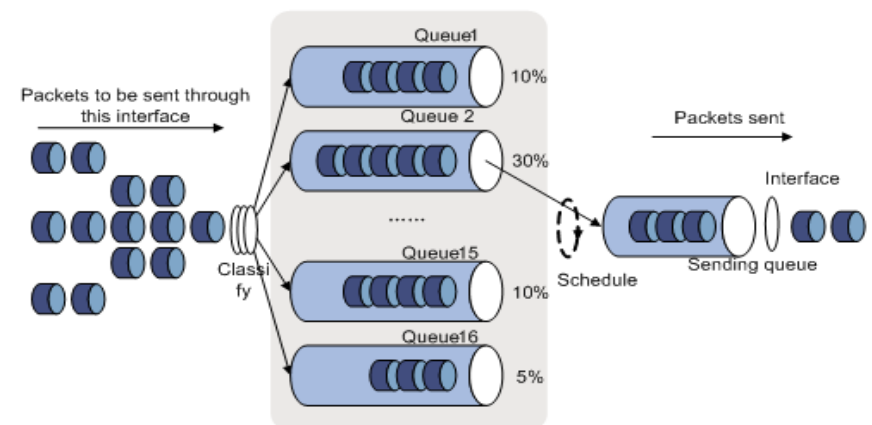

Fig 8: [25] SCF Scheduling Algorithm

SCF shows the higher performance when compared to WFQ \& DS with respect to queue management and resource utilization. In [20] SCF produced overall average for the throughput $110 \mathrm{Kbps}$ which is greater than WFQ. 


\subsubsection{Deficit Fair Priority Queuing (DFPQ)}

DFPQ with a counter was introduced to maintain the maximum allowable bandwidth for each service class. The counter decreases according to the size of the packets. The scheduler moves to another class once the counter falls to zero. DFPQ has also been used for inter-class scheduling. The problem with which DFPQ was introduced because queue length can be also used to set the priority level, e.g., more bandwidth is allocated to connections with longer queues. The direct negative effect of priority is that it may starve some connections of lower priority service classes. The throughput can be lower due to increased number of missed deadlines for the lower service classes' traffic. [16] In DFPQ and SS-assisted algorithms, the average end-toend delay for real-time packets increases at the beginning of the simulation time to its maximum value and then decreases to become almost stable. This initial increase is due to the fact that during the beginning of the simulation time, the SSs and the BS are busy attempting to complete the ranging process; hence, arriving packets are delayed causing a relatively high average delay. It is also observed that the DFPQ scheduler yields a higher average end-to-end delay for real-time classes than SSassisted algorithms. Indeed, DFPQ only focuses on achieving high bandwidth utilization by dynamically dividing the bandwidth between the UL and DL sub frames.

\subsection{Hybrid schedulers}

\subsubsection{TRS+RR scheduler}

It is the hybrid scheduling algorithm in which Temporary Removal Scheduler is combined with Round Robin to obtain the desired results. In [26] it involves identifying the packet call power, depending on radio conditions, and then temporarily removing them from a scheduling list for a certain adjustable time period TR. The scheduling list contains all the SSs that can be served at the next frame. When TR expires, the temporarily removed packet is checked again. If an improvement is observed in the radio channel, the packet could be topped up in the scheduling list again, otherwise the process is repeated for another TR duration. In poor radio conditions, the whole process could be repeated up to $L$ times at the end of which, the removed packed is added to the scheduling list, independently of the current channel condition. TRS+RR scheduler serves the highest number of SSs simultaneously in the same frame. Indeed, the TRS+RR scheduler serves all the SSs that belong to the scheduling list. In other words, the TRS+RR scheduler serves at least all the SSs having an SIR greater than a preset threshold. [13] From the results obtained TRS+RR throughput is greater than WFQ \& WRR.

\subsubsection{TRS+mSIR Scheduler}

TRS is combined with mSIR scheduler so that all the radio resources are reserved for the subscribers having the highest values of SIR. In [24], TRS+mSIR schedulers have good performance and deliver the highest number of packets. Indeed, these schedulers favor the SSs having the highest SIR values and then using the most efficient MCSs. TRS+mSIR schedulers have good performance and deliver the highest number of packets. Also due to the freezing of traffic of SSs having a small SIR, TRS+mSIR require a large average delay to deliver a data frame.

\subsection{3 $W R R+P Q$}

This hybrid scheduler from [4] ,use two types of scheduler: - Priority Queuing (PQ): In this scheduler, each queue has a priority. A queue can be served only if all higher priority queues are empty.
- Weighted Round Robin (WRR): In this discipline, each queue has a weight which defines the maximum number of packets that can be served during each scheduler round. This scheduler handles differently real time and non real time traffic: In the first stage, each traffic class is associated to a queue. This stage guarantees a fixed bandwidth for UGS and ErtPS classes and a minimum bandwidth for rtPS while ensuring fairness between flows because the rtPS packets have variable size and this flow could monopolize the server if the traffic is composed by packets with larger size than those of Class 1 and 2. In the second stage, output of the two WRR schedulers are enqueued in two queues F1 and F2, packets of these queues are managed by a priority PQ scheduler which gives higher priority to real time stream (stored in F1) which are more constringent in term of throughput and delay than the non-real time traffic (stored in F2) which are less time sensitive. The simulation results from [4] is the combination that it is recommended is to use TP as a selection traffic granularity method with MAXSNR as a mapping slot strategy after processing traffic by proposed hybrid scheduling block.

\subsubsection{WDRR+SP}

In this hybrid scheduler it uses two homogenous schedulers WDRR and SP. In the first stage we use WDRR and then in second stage it uses SP. From the Figure 9 in the first stage we use either DRR/PF/WDRR but from [5] it is analyzed that WDRR shows the best result with SP at that stage.

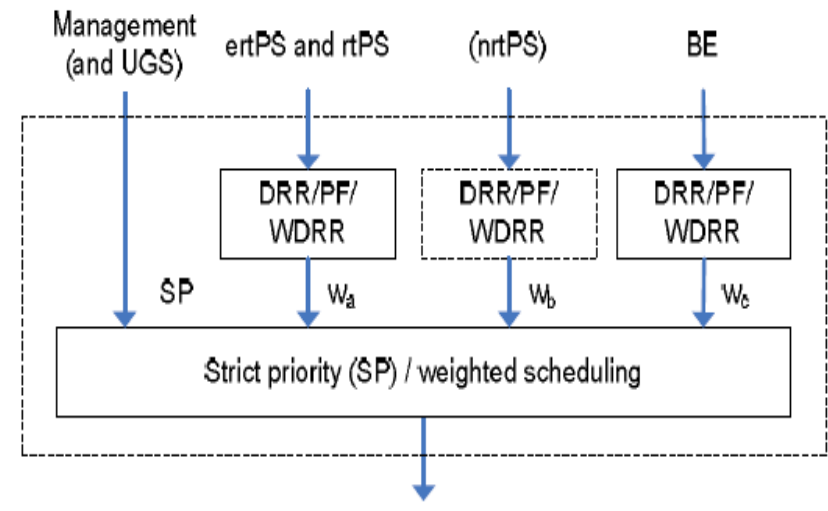

Fig 9: [5] A Hybrid WDRR + SP Scheduler

To avoid the starvation of BE connections as in SP, it is reserved a portion of all slots exclusively for these connections. Moreover, connection admission control should take care that there are always enough slots for the real-time connections.

It would easily enhance proposed scheduler and provide support for UGS and nrtPS classes as illustrated in Figure 9. For VoIP and other real-time traffic, DRR is still the best choice. It is not acceptable to let VoIP connections starve every now and then (when the most robust MCSs are used) just because that would lead into better MAC throughput. In fact, with PF scheduling, VoIP delay could grow intolerable if the number of VoIP connections is significant.

\subsubsection{EDF $+W F Q+F I F O$ Scheduler}

The hybrid algorithm proposed in [8] uses strict priority mechanism for overall bandwidth allocation and its complexity is $\mathrm{O}(\mathrm{N})$. The EDF scheduling algorithm is used for SSs of ertPS and rtPS classes, the WFQ algorithm is used for SSs of nrtPS class and FIFO is used for SSs of BE class. The EDF and WFQ algorithms are implemented as described in this homogenous algorithms section. FIFO is used for BE class as SSs of this class do not have any QoS requirements. This algorithm provides strict priority to ertPS and rtPS SSs; it results in a higher average throughput for the nrtPS class than the EDF algorithm. Also 
results in starvation of SSs of the BE class due to the strict priority nature of the algorithm and provide high priority to rtPS SSs, all the data of rtPS SSs will be flushed out in a frame. Under this algorithm when the concentration of BE SSs is low, the intra-class fairness of the nrtPS class is low and is high when the concentration of ertPS and rtPS SSs is high. This behavior of the algorithm is because it provides strict priority to nrtPS SSs over BE SSs.

\subsection{Opportunistic algorithms}

\subsubsection{Cross layer scheduler}

To manage resource allocation and grants an appropriate QoS per connection, other scheduling schemes are proposed and its complexity must be $\mathrm{O}(\mathrm{N})$. [18] These scheduling schemes rely on different algorithms to handle different classes of services for matching their QoS requirements. The fairness issue for the users in the same service class is also one of the important challenges to be considered. There are four service types are defined in IEEE 802.16e-2005 standard [11] which also have been mentioned in section 1.2, which includes UGS (Unsolicited Grant Service), rtPS (Real-time Polling Service), nrtPS (Non Real-time Polling Service), and BE (Best Effort). The UGS is designed to support real-time service flow that generates fixedsize data periodically, such as T1/E1 and VoIP without silence suppression. On the other hand, the rtPS supports the same with variable data size, such as video streaming services. Similarly, the nrtPS deals with FTP. The BE and ertPS perform tasks related to e-mail and VoIP respectively. The guaranteed delay aspect is taken utmost care in video streaming and VoIP. In the mobile WiMAX environment, the handover procedure begins as soon as the mobile SS moves into the service range of another BS. At physical layer, OFDMA is similar to OFDM using multiple sub-carriers to transmit data. However, while OFDM uses all available sub-carriers in each transmission, different sub-carriers could be arranged to different subscribers in downlink and each transmission could use the available subcarriers in uplink in OFDMA. There are three major steps involved in the Cross Layered approach. A simplified cross layered approach is shown figure 3. CAC algorithm takes the proper decision to admit or reject an incoming connection request along with its required bandwidth. Cross layer adaptations are essential for guaranteeing QoS supports in realtime multimedia traffic over wireless networks. In [11],[18] \& [26] literatures, many researchers have proposed various cross layer mechanisms for providing better QoS support to the system, but so far no such comprehensive cross layer design considering the parameters stated above, has yet been reported in the literature. [9] The cross layer scheduling provide these: QoS Guarantee, Channel Quality is also considered in Scheduling, but it is Complex in implementation and All slots per frame are allocated to highest priority connection.

In [26], author proposed scheduler offers prescribed delay, and rate guarantees for real time and non real-time traffic; at the same time, it uses the wireless bandwidth efficiently by exploiting multiuser diversity among connections with different kinds of services. Furthermore, scheduler enjoys flexibility, scalability, and low implementation complexity. Performance of proposed scheduler was evaluated via simulations in the IEEE 802.16[2] standard setting, where the upper-bound $\beta \mathrm{rtPS}$, $\beta$ nrtPS, $\beta \mathrm{BE}$, and the delay guard time $\Delta \mathrm{Ti}$ were set heuristically. Their effects on performance are worthy of further research. Furthermore, our scheduler allocates all $\mathrm{Nr}$ time slots to one connection each time for simplicity; however, scheduling multiple connections each time may lead to better performance, which is under current investigation.

\section{SURVEY ANALYSIS}

For homogenous schedulers we can analyze from the survey that Weighted Round Robin Scheduler is that its performance for rtPS class is more than any other scheduler when we use WRR as homogenous scheduler (Fig. 11, 12, 13, 14, 15, 16, 17, 18, 20, $22,24 \& 25$ ), but failed to give the same output for hybrid scheduler due to dynamic, buffer length \& trade-off between the throughput and queue delay Fig 10 in the network.
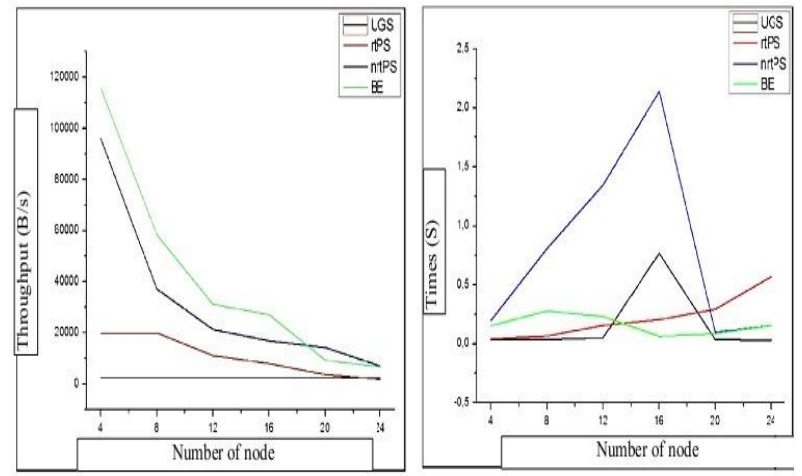

Fig 10: [21] Average Throughput and Average End to End Delay of WRR

WFQ shows the best performance as the average end-to-end time delay for UGS, rtPS, ertPs, B.E QoS classes but failed in nrtPS class also with average throughput Figure 11, 12, 13, 15, $16,17,18,20, \& 22$ and a superior performance compared to the WRR algorithm in the presence of variable size packets.

$\mathrm{RR}$ is the best scheduling algorithms with queue management and resource utilization than any other scheduling algorithms and best suited for less MSs (less than 50) Figure 11, 13, 15, 16, $21 \& 23$.

Figure from 11-14 show the average throughput of rtPS class.
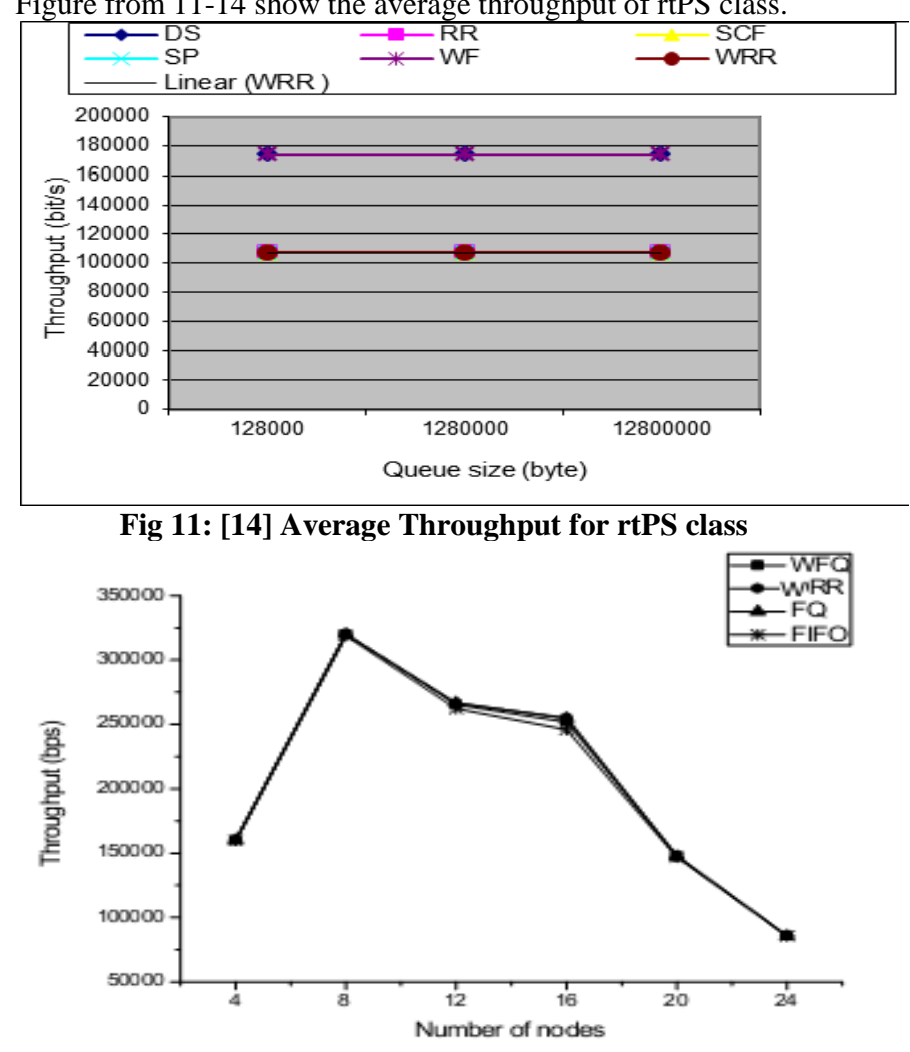

Fig 12: [21] Average Throughput for rtPS class 


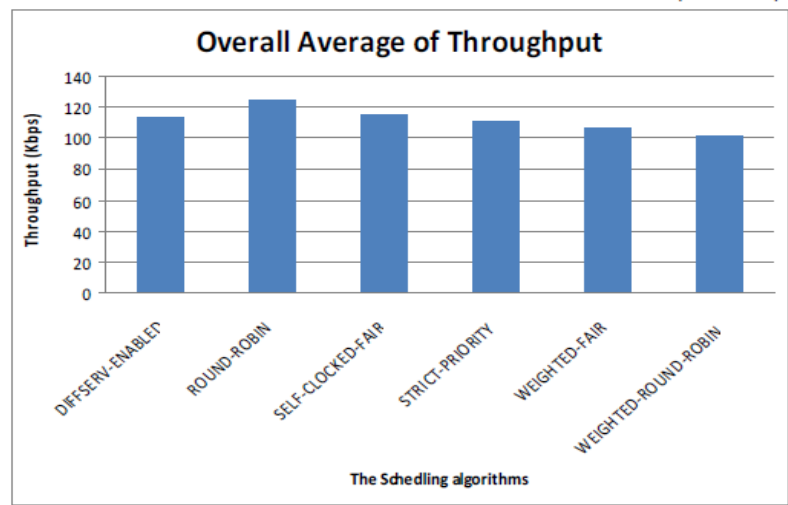

Fig 13: [20] Average Throughput for rtPS class

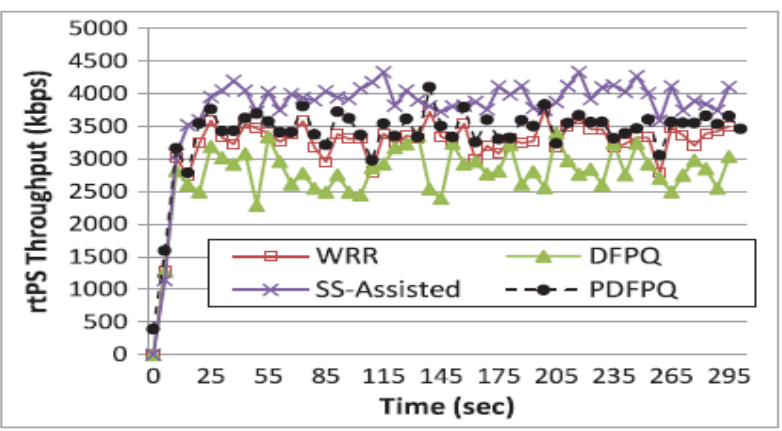

[16] Fig. 14 Throughput of rtPS class

Figures from 15-18 show the average end to end delay of the rtPS class.

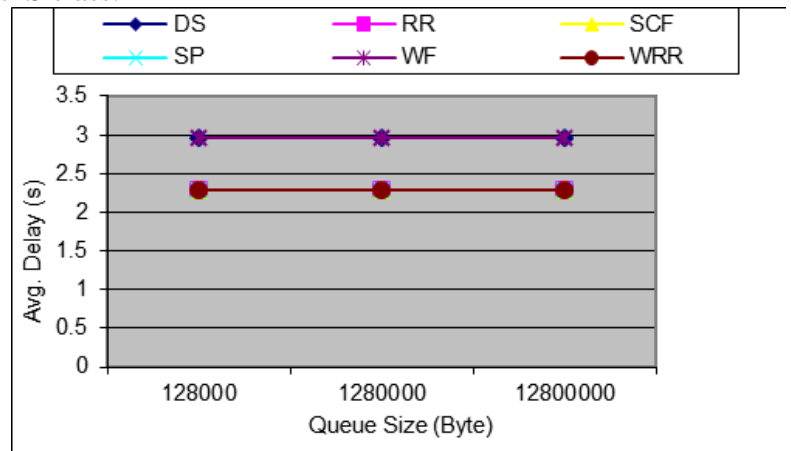

Fig 15: [14] Average Delay for rtPS class

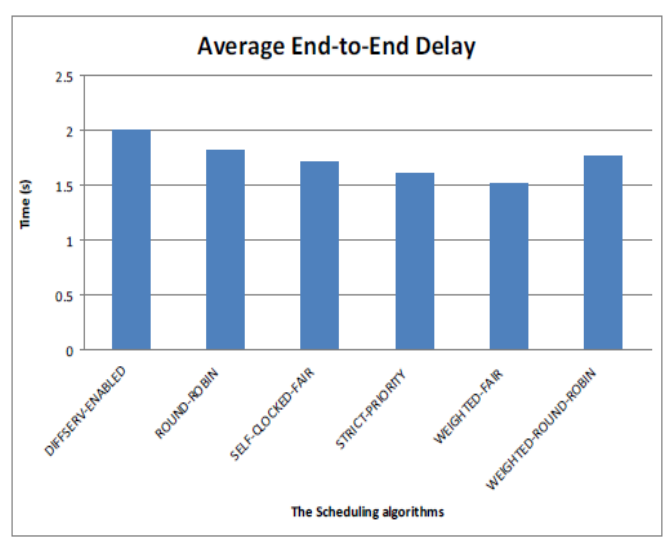

Fig 16: [20] Average End-to-End Delay for rtPS class

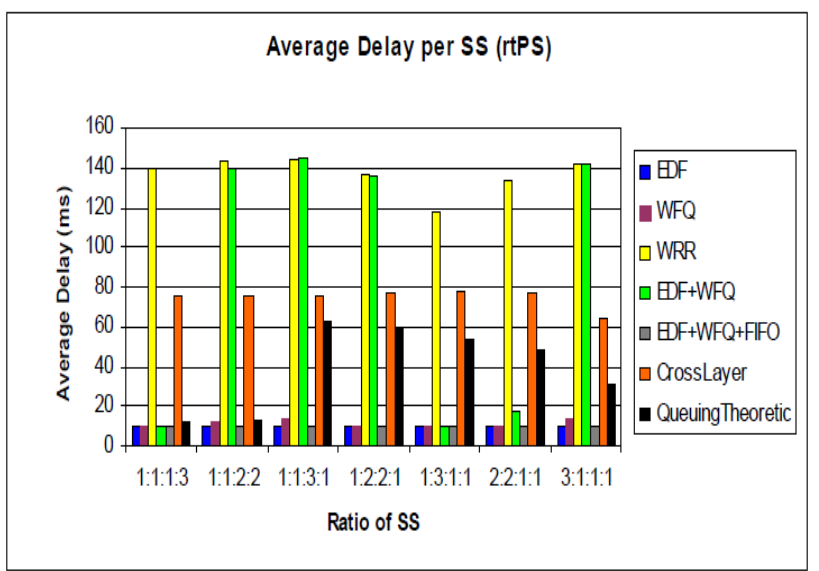

Fig 17: [8] Average Throughput for rtPS class.

EDF best suited for the ertPS or rtPS and provides average delay the since the algorithm provides strict priority to SSs with delay requirements and provides lower average throughput for the nrtPS Fig 17, 20, 22 \& 24

PF provides Fairness in Scheduling Priority Based Fig19, it is simple Implementation Multi-User Diversity Gain but failed to guarantee the QoS parameters. For real time traffic i.e., rtPS class DRR is still the best choice but it is not suited of UGS traffic Fig19.

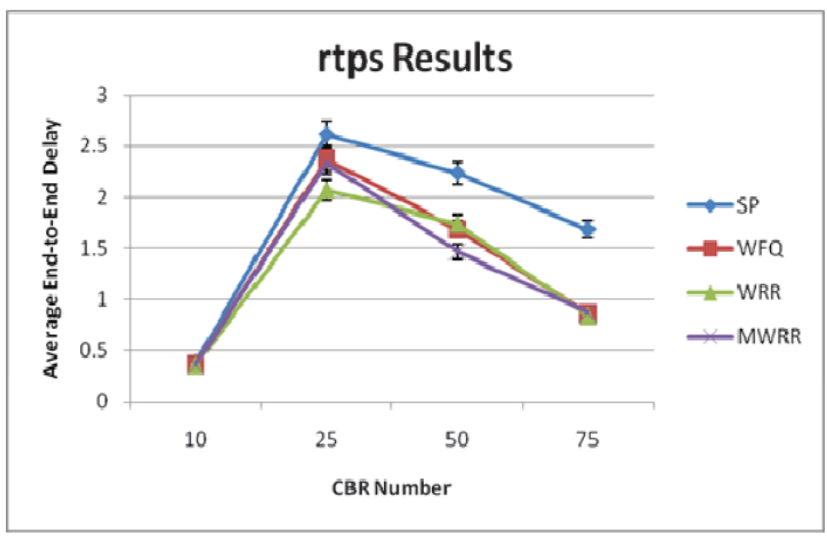

Fig 18: [17] End to End Delay rtPS class.

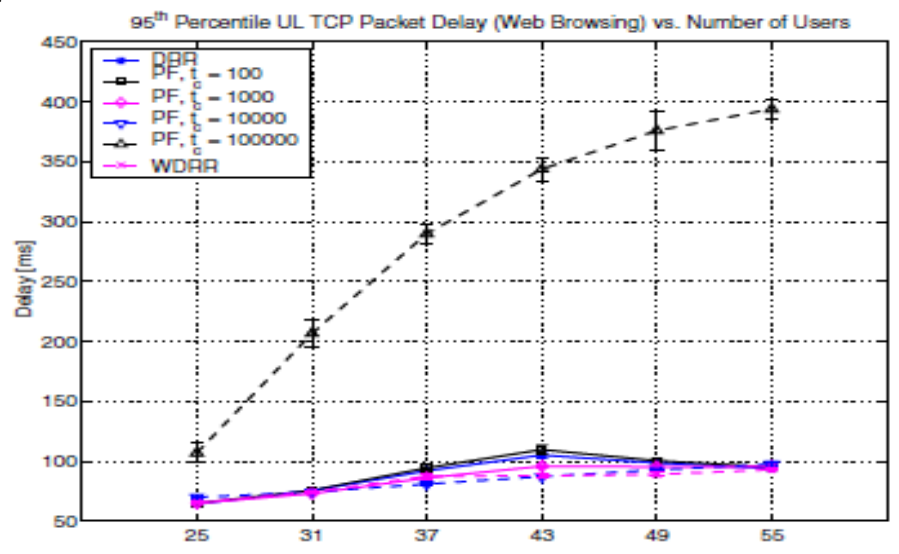

[5] Fig. 19. Fairness for rtPS class

WDRR seems to outperform PF when the number of connections is low, it could be feasible to combine PF and WDRR so that there would be a certain threshold after which the scheduling algorithm would change from WDRR to PF. DFPQ scheduler yields a higher average end-to-end delay for real-time 
classes than SS-assisted algorithms Fig14 .SCFQ can maintain the fairness attribute in virtual time function Fig. 11, 13, 15 \& 16.

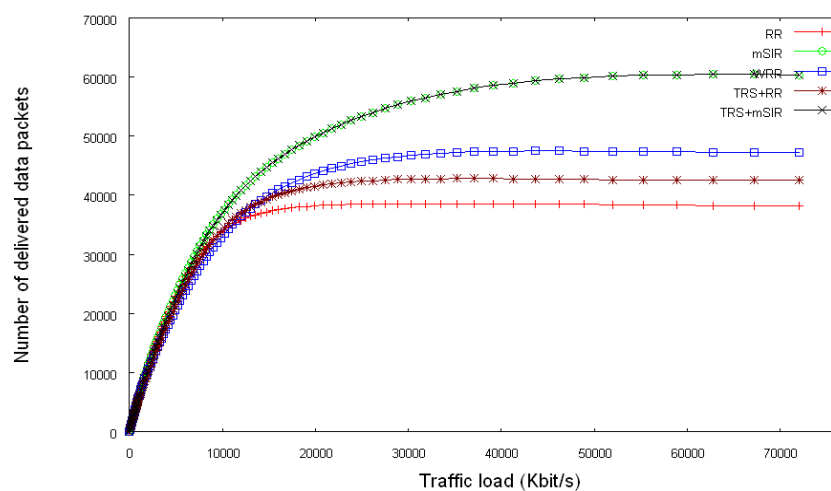

Fig 20: [24] Number of delivered data packets versus traffic load

\section{rtVR}

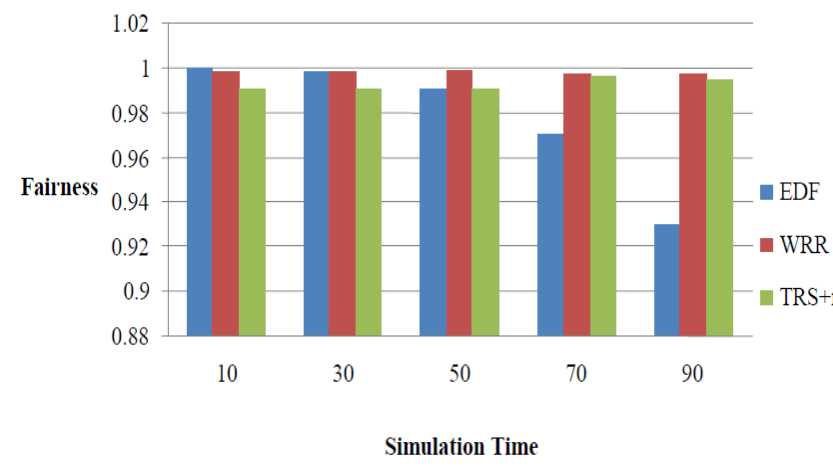

Fig 21: [13] Fairness for rtPS class

mSIR show a highly efficient utilization of radio resources. However, with the mSIR scheduler, the users with a SIR (Signal to Interference Ratio) that is always small may never be served (figure 21). DS is worst in case of queue management and resource utilization but proved a good algorithm with respect to throughput and delay Fig 11, 13 \& 15.

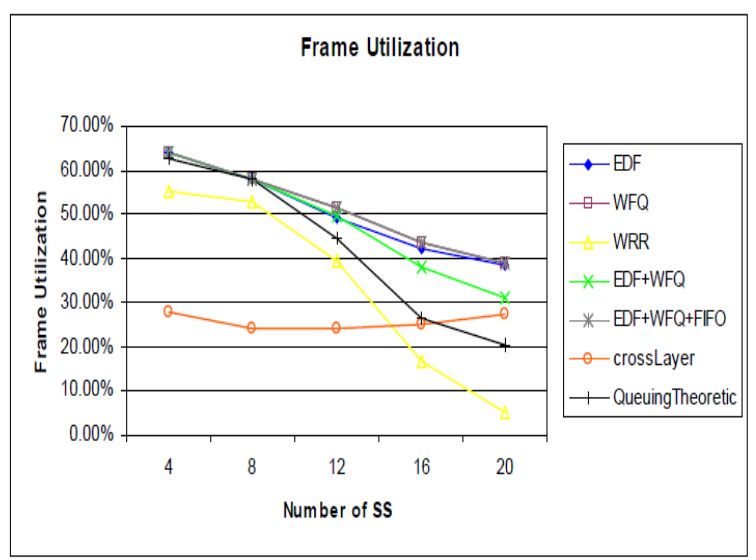

Fig 22: [8] Frame Utilization

Coming to the hybrid schedulers TRS+RR scheduler provides a best performance for real time classes like UGS \& rtPS traffic but an average for other classes Fig21. TRS+mSIR require a large average delay to deliver a data frame due to the freezing of traffic of SSs having a small SIR Fig 20 \& 21. WDRR combining with SP gives the feasible solution when the connections are low. EDF+WFQ+FIFO Scheduler perform low with $\mathrm{BE}$ QoS class and when the concentration of BE SSs is low, the intra-class fairness of the nrtPS class is low and is high when the concentration of ertPS and rtPS SSs is high.

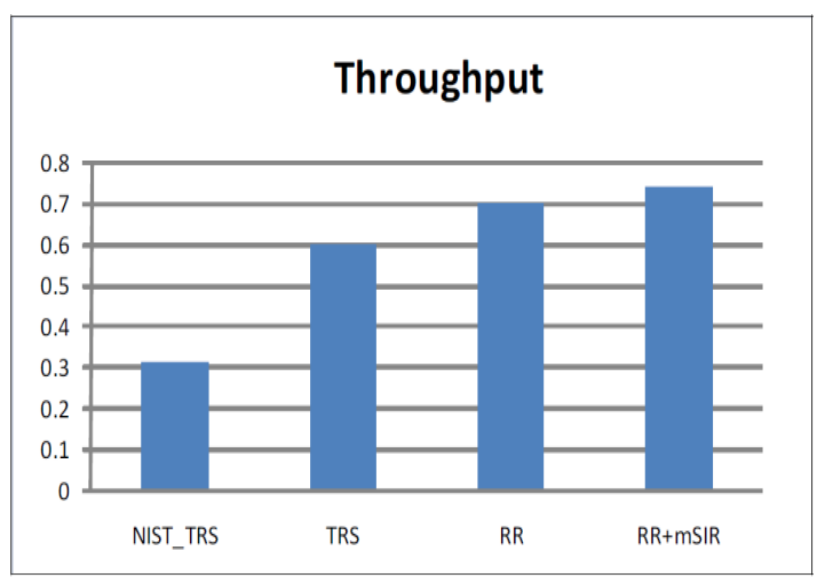

Fig 23: [13] Average Throughput for rtPS class

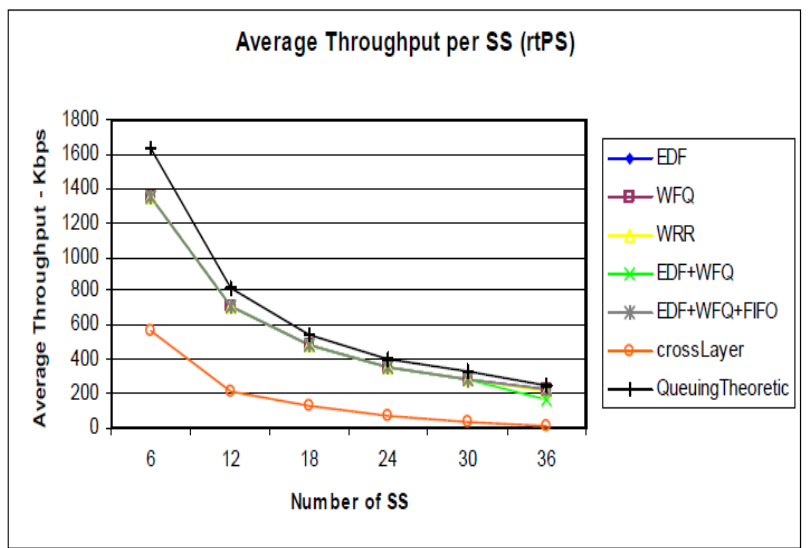

Fig 24: [8] Average Throughput for rtPS class

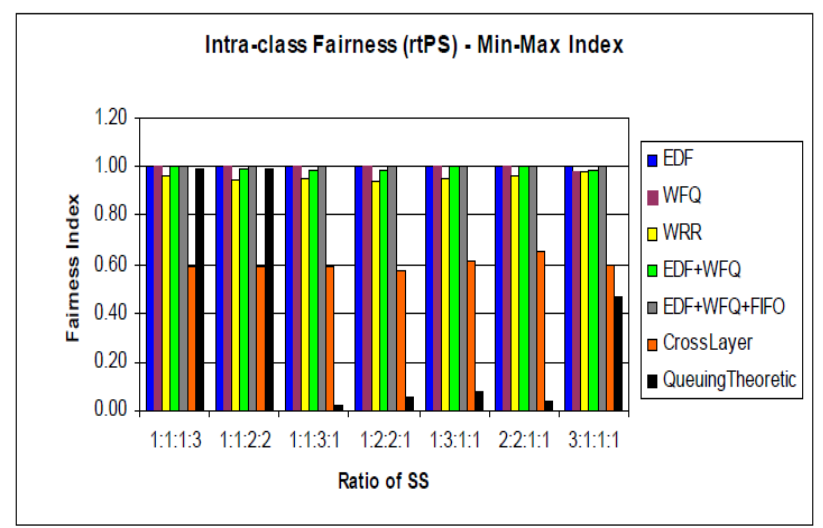

[8] Fig. 25. Intra-class Fairness for rtPS class

Now for the opportunistic schedulers, TCP-Aware Uplink Scheduling Algorithm provides an efficient utilization of resources among $\mathrm{BE}$ connections but it treats only one class and also complex in implementation. The cross layer considers fairness issue for the users in the same service class is also one of the important to handle the rtPS class (figure 17, 22, 24 \& 25). 


\section{CONCLUSION}

In this paper, WiMAX networks incorporate several qualities of service (QoS) mechanisms at the Media Access Control (MAC) level for guaranteed services for data, voice and video.

In the first section we have dealt with the IEEE 802.16e standard and its QoS mechanisms. Also we have studied the QoS traffic classes. In the second section we learnt about taxonomy of the schedulers and studied different types of schedulers.

In the third Section we dealt with survey of the papers of several authors in which they deal with the scheduling algorithms and the implementation. We compared the results of algorithm of different authors and in section 4 we analyzed the survey of the literature.

Coming to the conclusion, we can conclude that there are homogenous algorithms like WFQ, WRR, DS, SCFQ which can have a better performance but not for all the QoS classes. WRR is good for better throughput, WFQ is best for end to end delay and real-time classes (UGS, rtPS and ertPS), PF and TCP Aware Uplink algorithm are best suited for BE QoS class, RR algorithm was the best in terms of packet latency (Jitter), SP is not suited for multimedia data and DFPQ for achieving higher bandwidth utilization. To satisfy all the QoS traffic classes homogenous alone cannot handle the traffic, so we must have a hybrid or opportunistic schedulers (cross layer scheduler) to achieve the goals of IEEE 802.16 e QoS standard at MAC layer.

\section{REFERENCES}

[1] Chakchai So, Abdel-Karim Tamimi, Raj Jain, "Scheduling in IEEE 802.16e Mobile WiMAX Networks: Key Issues and a Survey" , JOURNAL ON SELECTED AREAS IN COMMUNICATIONS, VOL. 27, NO. 2, FEBRUARY 2009.

[2] G.S. Paschos, I. Papapanagiotou, C.G. Argyropoulos ,S.A. Kotsopoulos, "A Heuristic Strategy for IEEE 802.16 WiMAX scheduler for Quality of Service", Wireless Telecommunication Laboratory Electrical and Computer Engineering University of Patras Kato Kastritsi, 26500 Greece .

[3] Reena Dadhich, GeetikaNarang, D.M.Yadav International Journal of Engineering and Advanced Technology (IJEAT) ISSN: 2249 - 8958, Volume-1, Issue-3, February 2012167 Analysis and Literature Review of IEEE 802.16e (Mobile WiMAX) Security, 2012.

[4] Sondes Khemiri Guy Pujolle, Khaled Boussetta Nadjib Achir, "A Cross-Layer Radio Resource Management in WiMAX Systems ", Edited by Dr. Roberto Hincapie , ISBN 978-953-307-956-1 , Publisher InTech, Published online 03, February, 2012, Published in print edition February, 2012

[5] Jani Lakkakorpi, Alexander Sayenko, Jani Moilanen, “ Comparison of different scheduling algorithms for WiMAX base station: Deficit RoundRobin vs. Proportional Fair vs. Weighted Deficit RoundRobin" , In: Proceedings of the 2008 IEEE Wireless Communications and Networking Conference (WCNC 2008). Las Vegas, Nevada, USA. 31 March 3 April 2008

[6] P.S.Revankar Head, Avinash S Kapse, Dr. W. Z. Gandhare, "Overview of a MAC Scheduling Algorithm for IEEE 802.16 Wireless Networks", International Journal of Computer Applications (0975 - 8887) Volume 2 - No.8, June 2010

[7] IEEE 802.16e-2005, "IEEE Standard for Local and Metropolitan Area Networks - Part 16: Air Interface for Fixed and Mobile Broadband Wireless Access Systems Amendment 2: Physical and Medium Access Control
Layers for Combined Fixed and Mobile Operation in Licensed Bands", February 2006.

[8] Pratik Dhrona, "A Performance Study of Uplink Scheduling Algorithms in Point to Multipoint WiMAX Networks" A thesis submitted to the School of Computing in conformity with the requirements for the degree of Master of Science Queen's University Kingston, Ontario, Canada December, 2007.

[9] Ashish Jain, Anil K. Verma, "Comparative Study of Scheduling Algorithms for WiMAX”, 2008.

[10] Abhishek Maheshwari, "Implementation and Evaluation of a MAC Scheduling Architecture for IEEE 802.16 WirelessMANs" A thesis presented to the Indian Institute of Technology Kanpur in fulfillment of the thesis requirement for the degrees of Bachelor of Technology and Master of Technology in Department of Computer Science and Engineering IIT Kanpur, India, May 2006.

[11] Prasun Chowdhury, Iti Saha Misra, Salil K Sanyal, “ Cross Layer QoS Support Architecture with Integrated CAC and Scheduling Algorithms for WiMAX BWA Networks", (IJACSA) International Journal of Advanced Computer Science and Applications, Vol. 3, No. 1, 2012.

[12] N. Nikopoulos, "WIMAX MAC SCHEDULER DESIGN \& EXTENSIBLE SIMULATION FRAMEWORK FOR NETWORK SIMULATOR (NS-2)", Submitted in partial fulfillment of the requirements for the degree of Master of Science in Information Networking (MSIN) in the Information Networking Institute of Carnegie Mellon University at Athens Information Technology, Athens, Greece, 2008.

[13] Majid Taghipoor, Saeid MJafari, Vahid Hosseini "Scheduling Algorithm and Bandwidth Allocation in WiMAX", Edited by Dr. Roberto Hincapie, ISBN 978-953307-956-1, Publisher InTech, Published online 03, February, 2012, Published in print edition February, 2012.

[14] Ala'a Z. Al-Howaide, Ahmad S. Doulat, Yaser M. Khamayseh "PERFORMANCE EVALUATION OF DIFFERENT SCHEDULING ALGORITHMS IN WIMAX", Published in 2012.

[15] Gajanan M. Galshetwar, Prof. Amutha Jayakumar ,Yashika Mittal " Comparative Study Of Different Scheduling Algorithms For Wimax MAC Scheduler Design", International Journal of Engineering Research and Applications (IJERA) ISSN: 2248-9622 www.ijera.com Vol. 2, Issue 2, pp.1031-1037, Mar-Apr 2012,

[16] Haidar Safa, Samar Khayat, "A preemption-based scheduling algorithm for WiMAX networks", WIRELESS COMMUNICATIONS AND MOBILE COMPUTING Wirel. Commun. Mob. Comput. (2013) Published online in Wiley Online Library (wileyonlinelibrary.com). DOI: 10.1002/wcm.2368, 2013.

[17] Wail Mardini , Mai M. Abu Alfoul , “ Modified WRR Scheduling Algorithm for WiMAX Networks", Network Protocols and Algorithms, ISSN 1943-3581 2011, Vol. 3 No. 2 , Accepted: July 24, 2011 DOI: 10.5296/ npa.v3i2.751, 2011.

[18] Ronak Farhadi, Vahid Tabataba Vakili, Shahriar Shirvani Moghaddam, " A Novel Cross-Layer Scheduling Algorithm for OFDMA-Based WiMAX Networks”, Int. J. Communications, Network and System Sciences, 2011, 4, 98-103, doi:10.4236/ijens.2011.42012 Published Online February 2011 (http://www.SciRP.org/journal/ijens), 2011.

[19] Kim, Seungwoon, Yeom, Ikjun, "TCP-aware Uplimk scheduling for IEEE 802.16". IEEE Communication Letter, Feb., 2007.

[20] Mohammed Sabri Arhaif , "Comparative Study of scheduling Algorithms in WiMAX" International Journal of 
Scientific \& Engineering Research, Volume 2, Issue 2, February-2011.

[21] Hattab Guesmi , Sassi Maaloul, “ A Cross-Layer Qos Based Scheduling Algorithm WRR Design in Wimax Base Stations ", American Journal of Electrical and Electronic Engineering, 2013, Vol. 1, No. 1, 1-9 Available online at http://pubs.sciepub.com/ajeee/1/1/1 , Science and Education Publishing DOI:10.12691/ajeee-1-1-1, 2013.

[22] Subramanyam Y, Venkateswarlu Y, "WiMAX Base Station Scheduling Algorithms", TCS white paper, 2009.

[23] Dzmitry Kliazovich, rue Coudenhove Kalergi, "CROSS LAYER DESIGNS IN WLAN SYSTEMS", Edited by Prof. Nizar Zorba, Charalambos Skianis, Christos Verikoukis.

[24] Aymen Belghith, Loutfi Nuaymi, "Comparison of WiMAX scheduling algorithms and proposals for the rtPS QoS class".

[25] Operation Manual - QoS H3C S7500 Series Ethernet Switches,
http://www.h3c.com/portal/Technical_Support__Docume nts/Technical_Documents/Switches/H3C_S7500_Series_S witches/Configuration/Operation_Manual/H3C_S7500_O M-(Release_3100_Series)(V1.04)/200707/207838_1285_0.htm\#_Toc172462801

[26] Qingwen Liu , Georgios B. Giannakis, "A Cross-Layer Scheduling Algorithm With QoS Support in Wireless Networks" IEEE TRANSACTIONS ON VEHICULAR TECHNOLOGY, VOL. 55, NO. 3, MAY 2006.

[27] H. K. Rath, A. Bhorkar, , V. Sharma, "An Opportunistic DRR (O-DRR) Uplink Scheduling Scheme for IEEE 802.16-based Broadband Wireless Networks", IETE, International Conference on Next Generation Networks (ICNGN), Mumbai, 9 February 2006.

[28] Amir Hosain, A Thesis on "Implementation of Deficit Round Robin Scheduling Algorithm" , Jodar Communication Networks Laboratory http://www.ensc.sfu.ca/research/cnl School of Engineering Science Simon Fraser University, December 9, 2002. 